University of Wollongong

Research Online

Faculty of Engineering and Information

Faculty of Engineering and Information

Sciences - Papers: Part B

Sciences

2018

Coupled effects of initial orientation scatter and grain-interaction to texture evolution: a crystal plasticity FE study

Hui Wang

University of Wollongong, huiw@uow.edu.au

Cheng Lu

University of Wollongong, chenglu@uow.edu.au

Anh Kiet Tieu

University of Wollongong, ktieu@uow.edu.au

Lihong Su

University of Wollongong, lihongsu@uow.edu.au

Guanyu Deng

University of Wollongong, gdeng@uow.edu.au

Follow this and additional works at: https://ro.uow.edu.au/eispapers1

Part of the Engineering Commons, and the Science and Technology Studies Commons

Research Online is the open access institutional repository for the University of Wollongong. For further information contact the UOW Library: research-pubs@uow.edu.au 


\title{
Coupled effects of initial orientation scatter and grain-interaction to texture evolution: a crystal plasticity FE study
}

\begin{abstract}
Grain orientation and neighbourhood are two main factors that determine the in-grain and global texture. The coupled effects of them to texture evolution has not been well understood. In this work, initial orientations scattered from exact Cube at four different levels $\left(2^{\circ}, 5^{\circ}, 20^{\circ}\right.$ and $\left.45^{\circ}\right)$ were developed, running from near single crystal to polycrystal, which yielded grain-interaction at various grades. A crystal plasticity finite element model was developed and the predicted texture after a $50 \%$ and $75 \%$ reduction has been validated by experimental observations of both single crystals and polycrystals. When the deviation angles are small, the global texture is similar to that in exact Cube, where crystal rotation about transverse direction is dominant. Initial orientations of large scatter and grains of strong interaction led to high crystal rotations and the formation of rolling texture. To study the grain-interaction to specific grains, the orientations of neighbouring grains were replaced. It was found that texture evolution in the region close to grain boundaries is sensitive to grain-interaction, while crystal rotations in the inner region are basically determined by the initial orientation.

\section{Disciplines}

Engineering | Science and Technology Studies

\section{Publication Details}

Wang, H., Lu, C., Tieu, A. Kiet., Su, L. \& Deng, G. (2019). Coupled effects of initial orientation scatter and grain-interaction to texture evolution: a crystal plasticity FE study. International Journal of Material Forming, 12 (1), 161-171.
\end{abstract}




\title{
Coupled effects of initial orientation scatter and grain-interaction to texture evolution: A crystal plasticity FEM study
}

\author{
Hui Wang, Cheng Lu* , A. Kiet Tieu, Lihong Su, Guanyu Deng \\ School of Mechanical, Materials and Mechatronic Engineering, University of Wollongong, \\ Wollongong, NSW 2522, Australia \\ E-mail address: chenglu@uow.edu.au (Cheng Lu).
}

\begin{abstract}
Grain orientation and neighbourhood are two main factors that determine the in-grain and global texture. The coupled effects of them to texture evolution has not been well understood. In this work, initial orientations scattered from exact Cube at four different levels $\left(2^{\circ}, 5^{\circ}, 20^{\circ}\right.$ and $\left.45^{\circ}\right)$ were developed, running from near single crystal to polycrystal, which yielded grain-interaction at various grades. A crystal plasticity finite element model was developed and the predicted texture after a $50 \%$ and $75 \%$ reduction has been validated by experimental observations of both single crystal and polycrystal. When the deviation angles are small, the global texture is similar to that in exact Cube, where crystal rotation about transverse direction is dominant. Initial orientations of large scatter and grains of strong interaction led to high crystal rotations and formation of rolling texture. To study the grain-interaction to specific grains, the orientations of neighbouring grains were replaced. It was found that texture evolution in the region close to grain boundaries is sensitive to grain-interaction, while crystal rotations in the inner region are basically determined by the initial orientation.
\end{abstract}

Keywords: crystal plasticity FEM; texture; polycrystalline; grain interaction;

\section{Introduction}

Plastic deformation induced crystallographic texture has been a subject of research for decades because of its consequences for the material properties and deformation behaviours [1-3]. Among the texture components, Cube $\left\{\begin{array}{lll}0 & 0 & 1\end{array}\right\}<1 \quad 0 \quad 0>$ is an considerably investigated orientation in FCC structured metals (e.g., [4-14]). It is accepted that this orientation is unstable under rolling or plane strain compression (PSC) process [4-6], since it locates in the 'divergent' region of Euler space. A small perturbation of the initial orientation would change the slip activity and crystal rotation. For single crystal after a $\sim 50 \%$ thickness reduction, crystallographic orientations mainly rotate about transverse direction (TD) for exact Cube $[4,7,8]$ and also for the initial orientation rotated about TD by $5^{\circ}$ (5TD) [9], but the rotation axis is not specific for initially spined around rolling direction (RD) at $5^{\circ}(5 R D)$ [10]. A small deviation angle of $1.5^{\circ}$ led to an asymmetrical distribution of texture after a large reduction $[11,12]$. Small deviation angles in single crystals were chosen intendedly to study the stability of Cube orientation during deformation in Refs. $[9,10,13]$, and they can also be caused by sample preparation and/or orientation measurement [11, 13, 14]. Additionally, a set of large deviation angles can be used to approximate recrystallized texture in FCC metals such as aluminium, since which is of polycrystalline structure and near Cube orientation dominance $[15,16]$. The effect of initial orientations scattered from ideal Cube at different levels to the deformation texture has not been systemically investigated [17-19] and this is the thrust of the current study. 
Compared to the experimental research, initial texture can be perfectly created in numerical studies. Texture modelling has become a powerful tool and numerous crystal plasticity models have been proposed, such as Taylor model, self-consistent model and crystal plasticity finite element (CPFE) model [3]. The deformation of grains is assumed to be the same as the macroscopic strain in the fully constrained (FC) Taylor model, while a grain is treated as an inclusion in a homogenized medium that is of the averaged properties of all grains in the self-consistent model [3]. Different from these two models, no homogenization is assumed in CPFE, in which the crystal plasticity (CP) constitutive law is incorporated into finite element (FE) framework, where FE serves as the boundary problem solver and CP model is taken into calculation at each integration point [20]. The equilibrium of stress and strain for all elements is achieved by basic principles of mechanics in each FE increment [3] and accordingly, the deformation and texture evolution are fully coupled during deformation. By the way, an obvious deficiency of CPFE is the huge computation time. CPFE is in principle regarded as the best model for simulating plastic deformation and texture evolution [3, 21], which is thus adopted in the present investigation.

Due to the advantages of CPFE, the coupled effect of crystal orientations (crystallographic coordinate system relative to the sample coordinate) and neighbouring grains (grain-interaction) to the deformation texture can be fully accessed by this 'full-field theory' [3], where the two factors play an important role in texture evolution. For the first factor, as previously mentioned near Cube orientations, the deformation texture is very sensitive to the initial orientation [1], which has been documented by many experiments $[10,22,23]$. As for the second one, studying grain-interaction has started becoming more prevalent by using full-field CP models such as CPFE and crystal plasticity fast Fourier transform (CPFFT) due to the advances in parallel computing [13, 19, 22-24]. It has been found in these studies that in-grain subdivision caused by grain-interaction poses a significant influence on the global texture [13, 22-24]. Grain subdivision is determined by the initial grain orientation and local interactions, and as it proceeds, different parts of the grain rotated into different directions depending on the neighbours [25]. The effect of grain interaction to the global texture, but not to a specific grain, was studied in Ref. [22] in a sense of statistical manner. The ingrain subdivision caused by grain interaction was studied in Refs. [22, 23]. One grain was represented by one element in Ref. [23], which would lead to too strong grain interaction and result in poor texture prediction [22, 26]. Only 12 grains were considered in Ref. [25], so the finding substantially relies on the initial orientations of them, lacking statistical stability. The roles that initial orientation and grain-interaction play in in-grain texture and macroscopic texture have not been well understood [25]. As pointed out by Oddershede et al [1], studies on coupled effect of initial orientations and grain-interaction to micro- and macro-texture are still absent. Moreover, the rotation axis that is of great contribution to understand texture evolution [7] has received even less attention [27].

In this study, four polycrystal models with various levels of scatter in initial orientations were developed, which would create grain-interaction at different degrees in intensity. Meanwhile, the initial orientations spread due to the scatter. The impact that they (initial orientation and graininteraction) posed on the in-grain texture (for specific grains) and global texture (for a statistical assessment) is the subject of this paper. Texture evolution at a $50 \%$ and $75 \%$ rolling reduction was predicted by CPFE. The prediction was validated by experimental observations of both single crystal and polycrystal metals. It is straightforward to compare polycrystal results between simulation and experiment. A study on single crystal can only produce findings about a specific orientation that this single crystal initially has. Here, a large number of grains with diverse initial orientations were used 
to obtain a stable statistic data, which would cover the orientations of these single crystals. More specifically, the deformation behaviours in a single crystal would be roughly found in a grain of this polycrystal model when the grain-interaction is limited. The grain interaction on the selected grains was examined by regenerating the starting texture of the other grains. The influence of initial deviation angles, rolling reductions and grain-interaction to texture evolution has been investigated.

\section{CPFE implementation and simulation model}

The crystal plasticity model in the current research follows the well-recognized kinematical scheme proposed by Asaro [28] and developed by Peirce [29, 30]. This model was implemented into the commercial FE code Abaqus/Standard ver.6.9-1 by user-defined material (UMAT) subroutine. Bassani-Wu hardening model [31,32], a rate-dependent hardening model, is employed, which is regarded as the best texture predictor after comparison of five different hardening models [33]. The material used in the simulation is aluminium of high purity, and the slip plane and direction are $\{11$ $1\}$ and $\langle 110\rangle$, respectively. The material parameters in the hardening model were evaluated by fitting the simulated stress-strain curve with the experimental measurement of single crystal aluminium under PSC. The details of CP theory, CPFE implementation, hardening model, and material parameters have been given in Ref. [34]. This CPFE model and the set of material parameters have been used to study texture evolution under different processes $[6,34,35]$ and accurate predictions have been obtained.

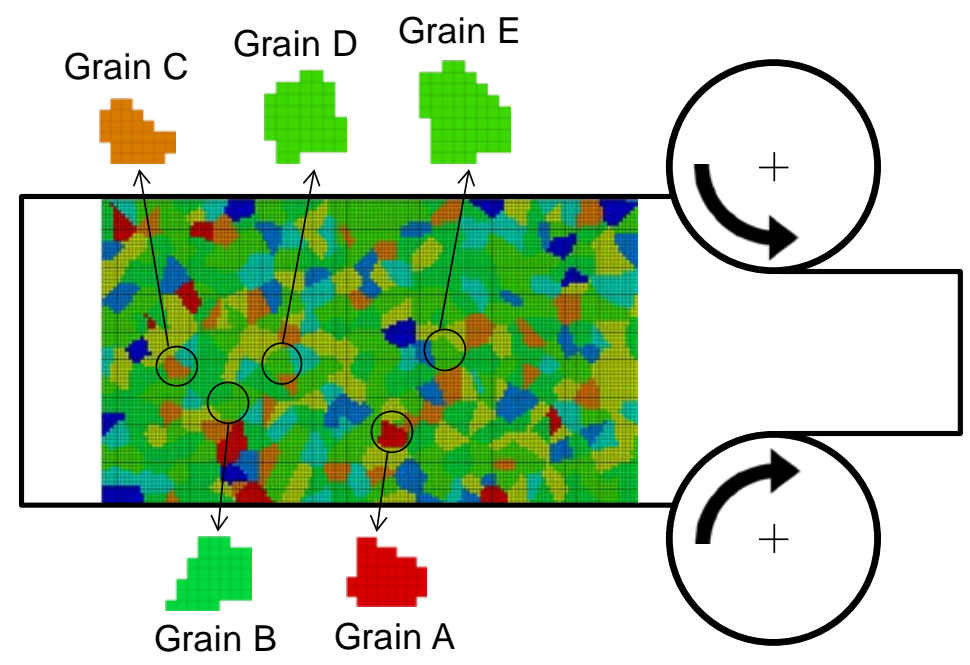

Fig.1. Rolling model with a deviation angle of $45^{\circ}$.

The simulation model in Fig.1 is two-dimensional (D) based on the assumption of plain strain deformation. The rolls were considered as analytical rigid bodies with a diameter of $125 \mathrm{~mm}$. To reduce convergence difficulties, the sheet $(2.0 \mathrm{~mm}$ in thickness) was placed with its leading end in the roll gap at the beginning of rolling. The sheet was driven to deform by rotating the rolls through the friction between the sheet and rolls, where a coefficient of friction of 0.1 was used to approximate the lubricated rolling condition [36]. The rolling was conducted in two passes (P) and the thickness reduction kept at $50 \%$ in each single pass, so the thickness after the first and second pass is $1 \mathrm{~mm}$ and $0.5 \mathrm{~mm}$, respectively, and the total reduction after the two passes is $75 \%$. The element type is CPE4R (a plane strain element with reduced integration point), which can provide 
efficient and fast numerical formulation. Enhanced hourglass control was applied to increase the resistance to the hourglassing problem and provide more accurate displacement solution. A part of the sheet was selected from the region of steady state of deformation, as shown in Fig.1, and then it was meshed into 11200 elements, and these elements were grouped into 375 grains to structure polycrystal model. On average, a grain includes 30 elements, so the in-grain texture is attainable. It is also polycrystal in the remainder of the sheet (its structure is not shown in Fig.1, but left blank), which was not taken into the analysis because of the effect of ends (not steady state of deformation). Four models with deviation angles of $2^{\circ}, 5^{\circ}, 20^{\circ}$ and $45^{\circ}$ from exact Cube were developed. To achieve an initial scatter, each of the three angles $\left(\operatorname{dev}_{\varphi_{1}}, \operatorname{dev}_{\phi}, \operatorname{dev}_{\varphi_{2}}\right)$ was chosen randomly from the region of the given deviation angles and then was superimposed to the Euler angles of ideal Cube $\left(\varphi_{1}=0, \phi=0, \varphi_{2}=0\right)$. Taking the deviation angle $5^{\circ}$ as an example, the three angles $\left(\operatorname{dev}_{\varphi_{1}}\right.$, $\left.\operatorname{dev}_{\phi}, \operatorname{dev}_{\varphi_{2}}\right)$ were selected at random in the region $\left[-5^{\circ}, 5^{\circ}\right]$ and then added to the three Euler angles of Cube, so the three Euler angles of one initial orientation are $\varphi_{1}=0+\operatorname{dev}_{\varphi_{1}}, \phi=0+$ $\operatorname{dev}_{\phi}, \varphi_{2}=0+\operatorname{dev}_{\varphi_{2}}$. The initial orientations were obtained by repeating the procedure up to $\sim 375$ times and they were assigned to the grains. All the elements in one grain have the same initial orientation.

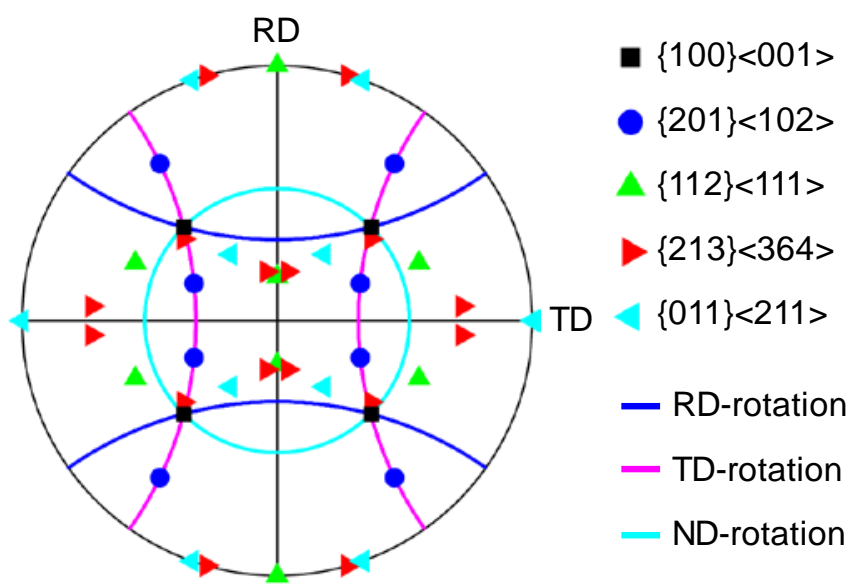

Fig.2. (1 111 ) pole figure shows the positions of five ideal crystal orientations and crystal rotation paths of RD-, TD- and ND-rotation from Cube.

In this study, (llll 111$)$ pole figures were used to present the starting and final texture. The positions of five ideal crystal orientations are shown in Fig.2. In the following, the crystal rotation in each element means the misorientation between the initial orientation (before rolling) and final orientation (after deformation), which is calculated according to Angle/Axis. This crystal rotation was further partitioned into three components representing rotations about the three axes, i.e., RD, TD and normal direction (ND), by following the method proposed by Wert et al. [37]. The rotation paths of pure RD-, TD- and ND-rotation from Cube are also illustrated in Fig.2. For instance, a pure TDrotation at $26^{\circ}$ would transfer Cube to $\left\{\begin{array}{lll}1 & 0 & 2\end{array}\right\}<2 \quad 01>$, while a combination of at least two from the three partitioned rotations (RD-, TD- and ND-rotation) is needed for Cube rotating to $\left\{\begin{array}{llllll}1 & 1 & 2\end{array}\right\}<1 \quad 1>$. 


\section{Results and validation}

In the following, pole figures and (partitioned) crystal rotation angels were used to characterize the texture evolution, where all the 11200 elements were taken into calculation. Experimental observation from lubricated rolling and PSC was used to validate the prediction. PSC can be used to approximate rolling when the shear strain is very low [36, 38, 39]. A small coefficient of friction (0.1) was used in the simulation, so the approximation is reasonably acceptable.

\subsection{Effect of deviation angles}

Fig.3a-c show the distribution of deformed FE meshes and crystal rotation angles in three models (deviation angles of $5^{\circ}, 20^{\circ}$ and $45^{\circ}$ ) after a $50 \%$ reduction, where for better contrast, larger rotation angles were reduced to be within the limit, while this reduction was not applied to d showing the deformed grains. The initially equiaxed grains were elongated along RD after rolling. The deformed FEM meshes are relatively homogeneous in Fig.3a, and become distorted with increasing deviation angles in $b$ and $c$. The deformation within grains is not homogeneous too (Fig.3d).

(a)

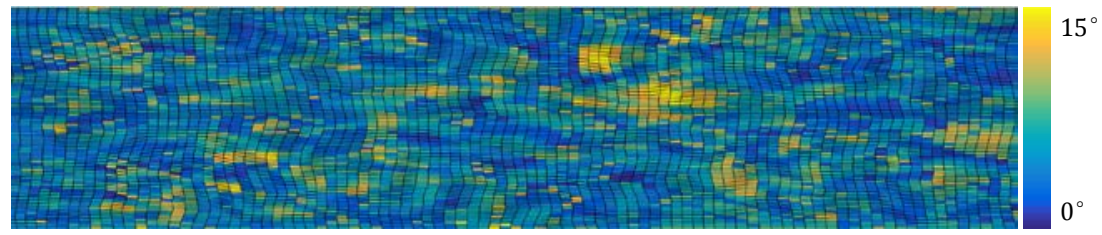

(b)

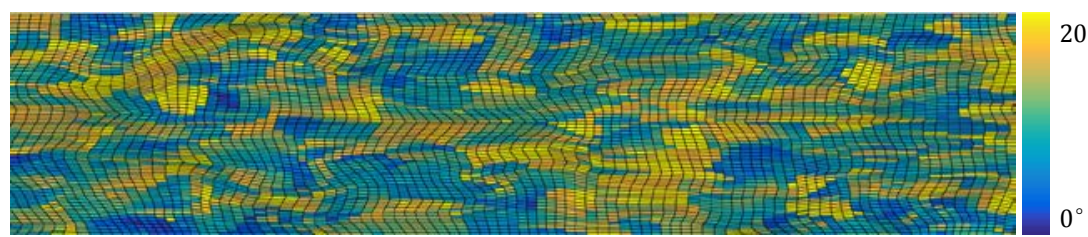

(c)

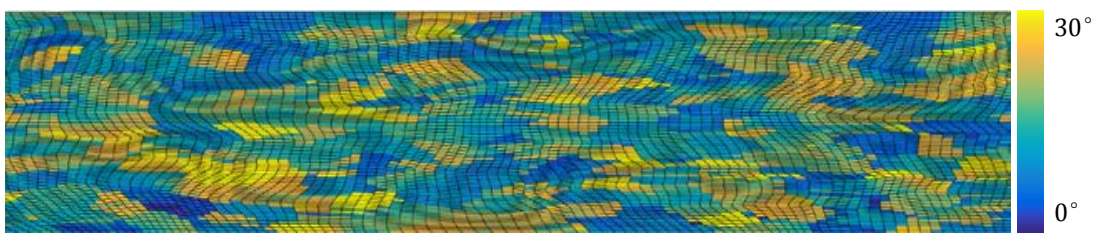

(d)
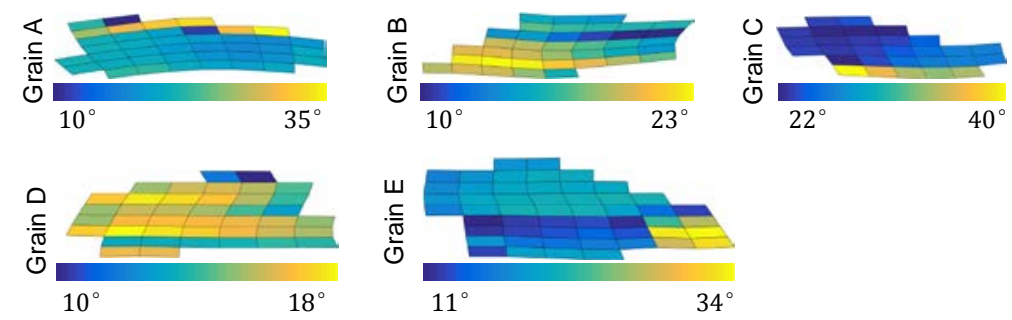

Fig.3. Distribution of deformed meshes and crystal rotation angles in models with deviation angles of (a) $5^{\circ}$, (b) $20^{\circ}$, (c) $45^{\circ}$, and (d) five selected grains (shown in Fig.1) after a $50 \%$ reduction. Larger rotation angles were reduced to be within the limit in a-c.

In Fig.4, the (1 $\left.\begin{array}{lll}1 & 1\end{array}\right)$ pole figures present the initial and final texture of the four simulations at a 50\% reduction. The initial orientations slightly scatter around Cube in the model with a $2^{\circ}$ deviation angle (Dev2) and after rolling, TD-rotation is highly dominant over RD- and ND-rotation. The predicted texture matches well with the experimental observation (Fig.4), in which an aluminium single crystal deviated from exact Cube by $\sim 1.5^{\circ}$ was cold rolled to a $50 \%$ reduction [11]. The maximum 
partitioned TD-rotation angle is $16.3^{\circ}$ in Dev2, which is close to the experimentally measured $15^{\circ}$ of exact Cube at the same strain [4]. The distribution of the three partitioned crystal rotation in Fig.5a shows that TD-rotation concentrates at below $10^{\circ}$, obviously higher than the other two components. The prediction in Dev2 is consistent with the experimental studies $[4,8,11,40]$ and crystal plasticity simulations $[2,6]$ of ideal Cube, so it can be said that the effect of a $2^{\circ}$ deviation angle is very trivial within a $50 \%$ reduction. However, it is not the case for Dev5. Though TD-rotation still dominates in the final texture (Fig.4 and $5 \mathrm{~b}$ ) with a maximum angle of $24.9^{\circ}$, small non-TD-rotations also developed. As for experimental studies (Fig.4), aluminium single crystal with an initial orientation rotated about TD by $5^{\circ}$ (5TD) has been deformed by PSC up to $60 \%$ reduction [9], and the main crystal rotation is about TD, while the rotation axes are TD and ND for 5ND at the same strain [9]. As for $5 \mathrm{RD}$, the rotation axis RD is also added to TD and ND at a $50 \%$ rolling reduction [10]. The direction of initial rotation is significant to the texture evolution when the deviation angle reaches $5^{\circ}$.

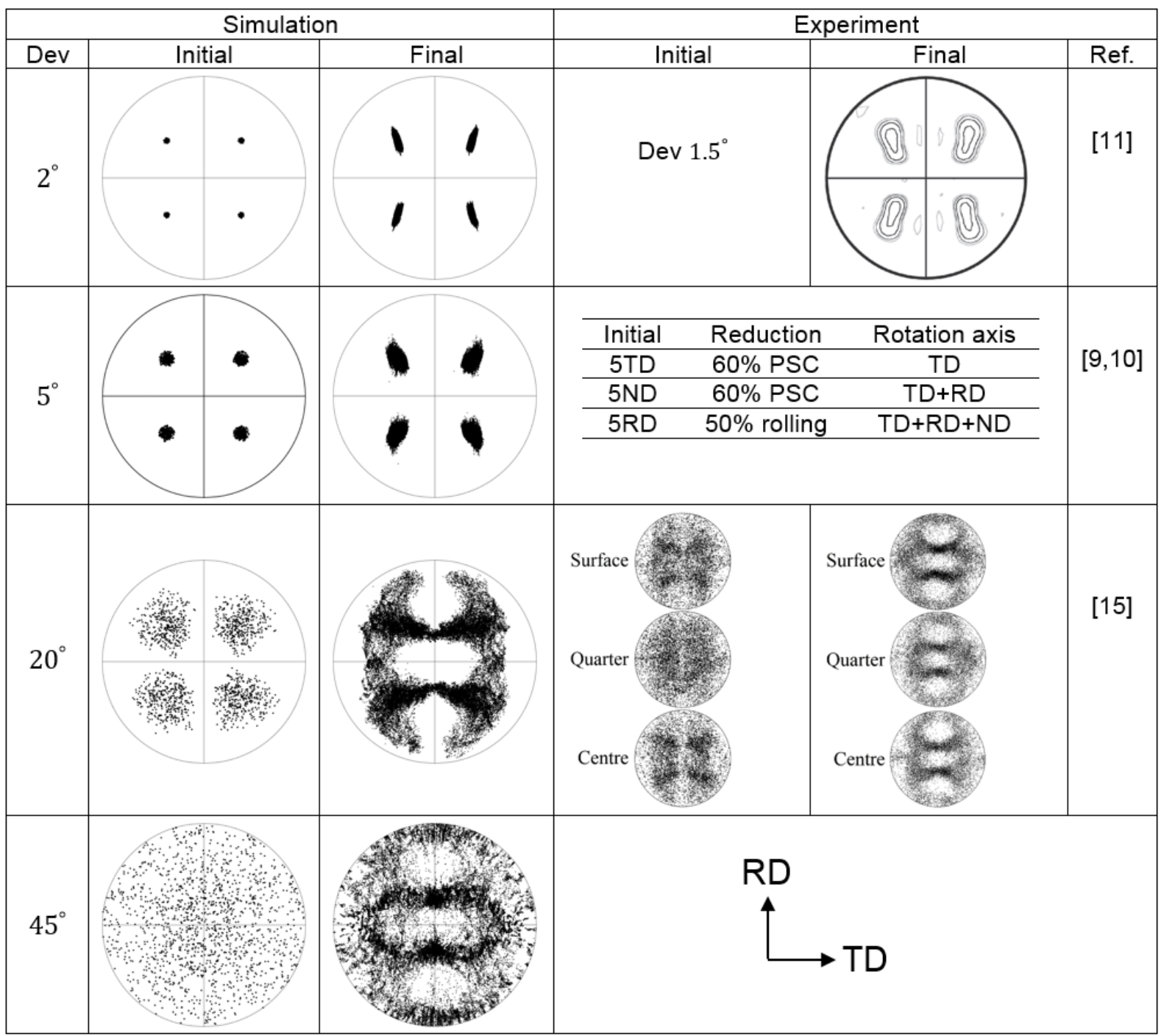

Fig.4. Comparison between simulated texture and experimental observation after a $50 \%$ reduction in all four models.

The initial texture of Dev20 is similar to the recrystallized texture in Fig.4 that was experimentally observed from the surface, quarter-thickness, and centre region [15]. The simulated texture trended

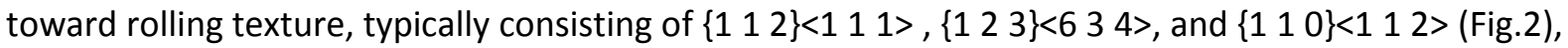


though it is still rudimentary, similar to the experimental result [15] (Fig.4). In Dev20, the partitioned RD- and ND-rotation increased quickly and are comparably large to TD-rotation (Fig.5c). A similar distribution of deformation texture (Fig.4) and partitioned rotations (not shown here) as that in Dev20 was also observed in Dev45, but the rolling texture intensity was promoted.
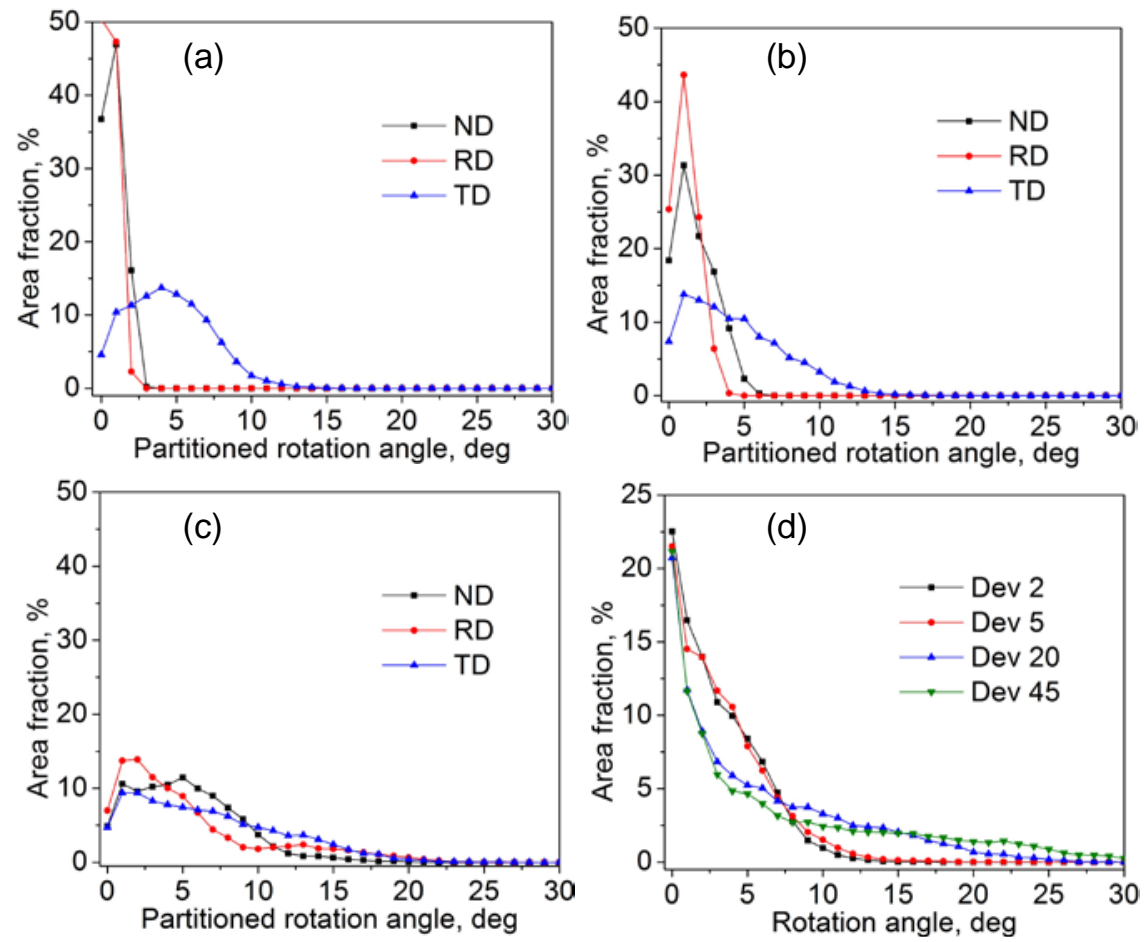

Fig.5. Distribution of ND-, RD-, and TD-rotation in models with deviation angles of (a) $2^{\circ}$, (b) $5^{\circ}$, and (c) $20^{\circ}$, and (d) crystal rotations in all four models at a $50 \%$ reduction.

The distribution of crystal rotation angles, i.e., misorientation between the initial and final orientations, of all elements in the four models is plotted in Fig.5d. Dev2 and Dev5 have a similar distribution, concentrating at below $12^{\circ}$. The fraction of large rotation angles $\left(>15^{\circ}\right)$ in Dev45 is noticeably higher than that in Dev20.

\subsection{Influence of rolling reductions}

Fig. 6 and 7 illustrate the simulated texture and distribution of partitioned rotations of three models $\left(2^{\circ}, 5^{\circ}\right.$, and $\left.20^{\circ}\right)$ after the second pass, respectively, where the partitioned rotations were calculated relative to the crystal orientations after the first pass, but not the initial orientations, i.e., rotations developed in the second pass alone, but not cumulative values. In Dev2, TD-rotation is still dominant (Fig.6) and non-TD-rotation appeared only in a few elements after their orientations reached about $\left\{\begin{array}{lll}1 & 0 & 2\end{array}\right\}<2 \quad 01>$ (shown in Fig.2). This observation is in agreement with the experimental observation in an aluminium single crystal of exact Cube after a 70\% rolling reduction [10] (Fig.6). TD-rotation over $10^{\circ}$ increased obviously (Fig.7a), even higher than that after the first pass (Fig.5a), while RDand ND-rotation remain very low. In Dev5, non-TD-rotation started to emerge, which is consistent with the experimental observation (Fig.6) that TD is not the only rotation axis anymore even for 5TD [9]. It seems that orientations tend to rolling texture, especially for large crystal rotations $\left(>15^{\circ}\right)$ (marked in red). It is interesting that the distribution of TD- and ND-rotation after the second pass (Fig.7b) is similar to that in the first pass (Fig.5b), while RD-rotation increased fast. In Dev20, the 
rolling texture was improved in intensity, especially for the large rotation angles $\left(>25^{\circ}\right)$ (marked in red). In this case, the RD-rotation slightly exceeded TD-rotation at relatively large rotation angles $\left(>7^{\circ}\right)$.

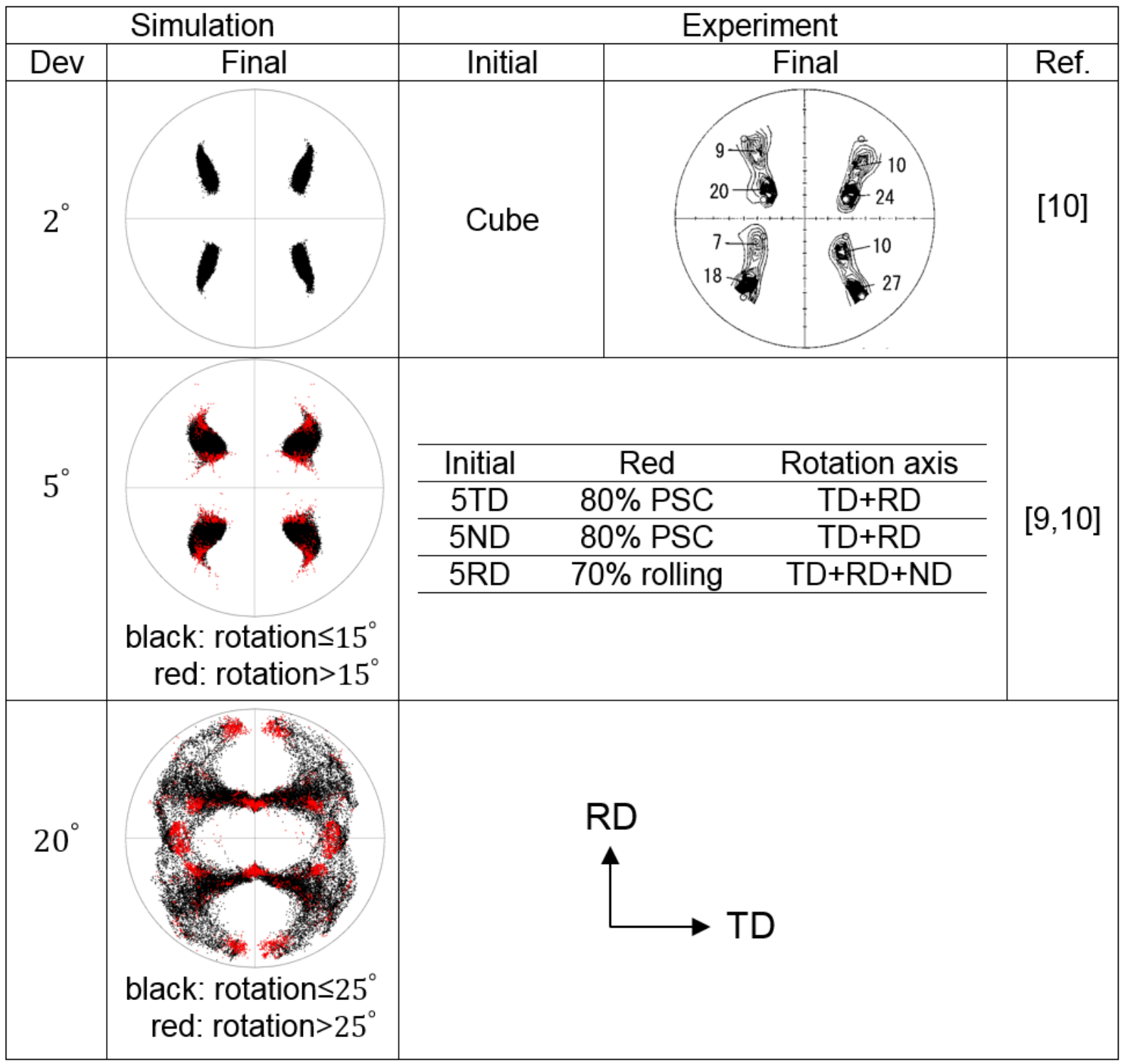

Fig.6. Comparison between simulated texture and experimental observation after a $75 \%$ reduction in three models.

For Dev5 in Fig.7d, it is surprising that the fraction of large crystal rotation angles $\left(>7^{\circ}\right)$ developed in the second pass alone, relative to that after the first pass, (Dev5, 2P to $1 \mathrm{P})$ is slightly higher than that developed in the first pass (Dev5, 1P to OP). This is to say the crystal orientations reached semistable positions by TD-rotation in the first pass, and after increasing the reduction to $75 \%$, large crystal rotations, especially RD-rotation, evolved. However, in Dev20 (Fig.7d), the fraction of relatively large crystal rotation angles $\left(>9^{\circ}\right.$ ) developed in the second pass (Dev20, $2 \mathrm{P}$ to $1 \mathrm{P}$ ) is lower than that after the first pass (Dev20, 1P to OP). This is because the crystal orientations rotated close to final stable positions (rolling texture) in the first pass and meanwhile, grain-interaction became weak, so the rotation rate decreased gradually [23, 24, 27]. This tendency is more pronounced in Dev45 (not shown here). 

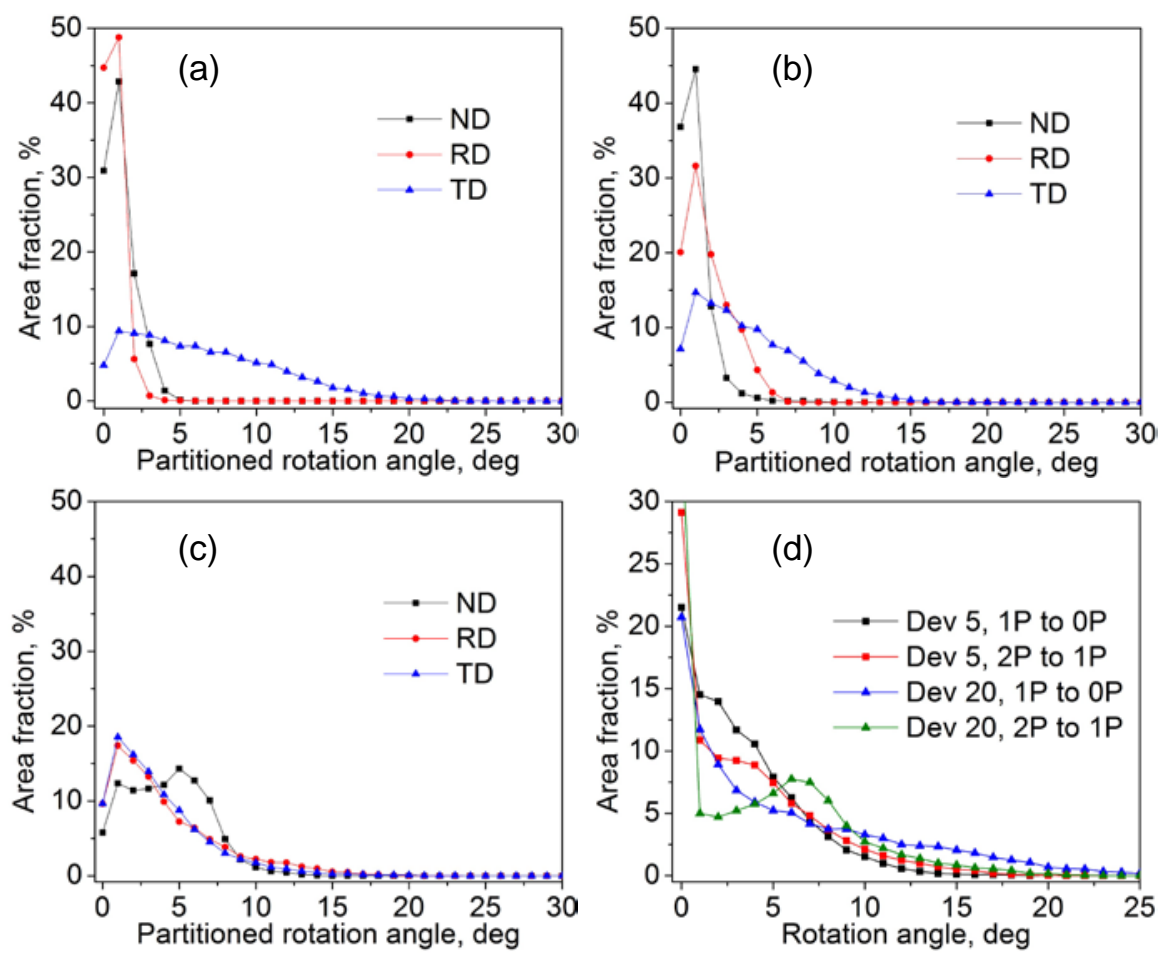

Fig.7. Distribution of partitioned ND-, RD-, and TD-rotation developed in the second pass alone (relative to the orientations after the first pass, but not initial orientations) with deviation angles of (a) $2^{\circ}$, (b) $5^{\circ}$, and (c) $20^{\circ}$, and (d) crystal rotations at a $75 \%$ reduction.

\subsection{Effect of crystal orientations and neighbouring grains}

The increasing deviation angles in these models lead to two consequences: the initial orientations of more randomness and misorientation angles between adjacent grains of large value. Their effect to in-grain texture was investigated in another simulation conducted for the model $45^{\circ}$ ( $50 \%$ reduction). Five grains, A-E, shown in Fig.1 were selected after rolling of the original polycrystal model, where the five grains are not contiguous with each other to avoid direct interaction among them. In this simulation, the polycrystal construction maintained. The initial orientations of the five selected grains kept unchanged, but the orientations of the other grains were regenerated following the method described in the Section 2. The simulation of the five grains was conducted up to three times by repeating this procedure of orientation replacement in neighbouring grains. The initial, final, and averaged final orientations of the selected grains are shown in red, black and blue in Fig.8, respectively. The averaged crystal rotation angles of each grain, relative to its initial orientation, in each model are listed in Table 1. 


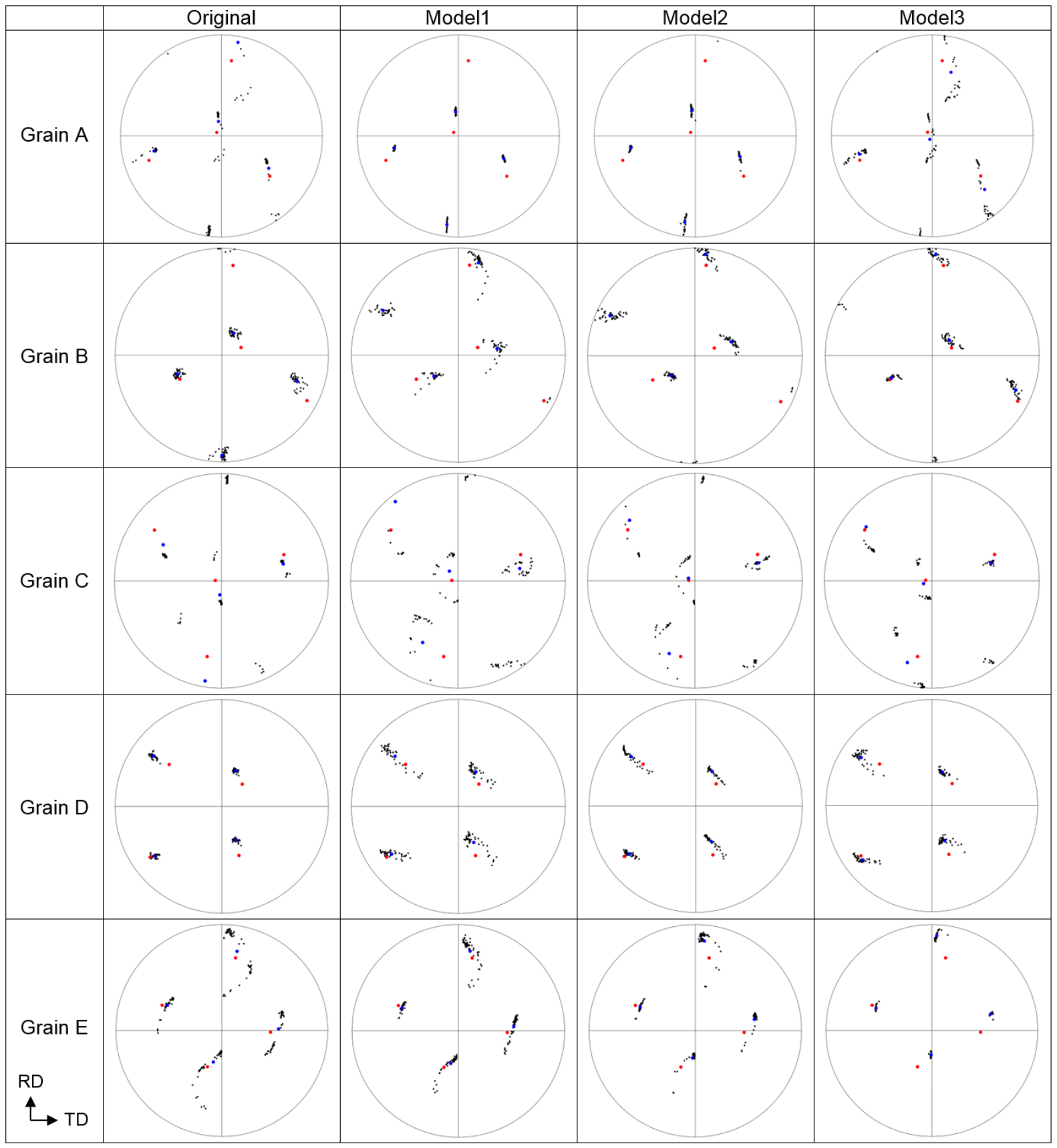

Fig.8. ( $\left.\begin{array}{lll}1 & 1 & 1\end{array}\right)$ pole figures of 5 selected grains, where the initial, final, and averaged final orientations are shown in red, black and blue, respectively.

For each individual grain, the developed texture is different from model to model due to the replacement of orientations in the other grains. The change of initial orientation would alter the slip system activation and plastic deformation, and then pose a different impact on the neighbouring grains. However, the variation of averaged rotation angles between models is not large in most grains, and the maximum change is only $11.6^{\circ}\left(=18.5^{\circ}-6.9^{\circ}\right.$, between Model 1 and Model3 in Grain E) in Table 1, which means grain interaction is limited even though the grain-interaction is large in Dev45. The severe grain-interaction is only limited to elements near grain boundaries (Fig.3d), especially at triple junctions, and large rotation angles evolved in these elements. These elements are small in number, corresponding to the minor texture components that are of large change in 
different models (Fig.8). In general, the rotation angles in the inner region of a grain are roughly at the degree of $15^{\circ}$ (Fig.3d), which are basically determined by the initial orientations. The inner elements contribute to the major texture components in Fig.8, and for a grain with different neighbourhood, the evolution of these major texture components is of similarity. Compared to grain-interaction, the initial orientation plays a basic and dominating role $[22,23,41]$. The graininteraction imposed to a grain is also influenced by the grain orientation [23], like Grain $D$, in which the variation of averaged rotation angles is very small (Table 1). The more unstable a orientations is, the more sensitive it is to the neighbourhood [23]. Though the number of elements bearing large rotation angles (caused by grain-interaction) in a grain is low, the crystal rotation change dramatically in direction and magnitude, so the variation of averaged rotation angles in Table 1 is not small.

Table 1. Averaged crystal rotation angles of five grains in different models.

\begin{tabular}{|c|c|c|c|c|}
\hline & Original & Model1 & Model2 & Model3 \\
\hline Grain A & $13.1^{\circ}$ & $23.1^{\circ}$ & $24.9^{\circ}$ & $11.9^{\circ}$ \\
\hline Grain B & $16.4^{\circ}$ & $19.2^{\circ}$ & $18.1^{\circ}$ & $8.3^{\circ}$ \\
\hline Grain C & $16^{\circ}$ & $18^{\circ}$ & $7.8^{\circ}$ & $7^{\circ}$ \\
\hline Grain D & $14.8^{\circ}$ & $12.7^{\circ}$ & $13.1^{\circ}$ & $15^{\circ}$ \\
\hline Grain E & $6.9^{\circ}$ & $7.4^{\circ}$ & $14.5^{\circ}$ & $18.5^{\circ}$ \\
\hline
\end{tabular}

\section{Discussion}

In this study, plain strain condition in the 2D model was used to approximate the real 3D experiment. It is true that 2D model is not capable of predicting strain out of plane, but RD- and ND-rotation on the TD-ND and RD-TD plane, respectively, were predicted. In the adopted CPFE model, crystal slip and lattice rotation are the two mechanisms for deformation. The material spin $\Omega$ represented by macroscopic shear strain is composed of two parts, $\boldsymbol{\Omega}^{\mathrm{p}}$ and $\boldsymbol{\Omega}^{\mathrm{e}}$, i.e.,

$$
\boldsymbol{\Omega}=\mathbf{\Omega}^{\mathrm{P}}+\mathbf{\Omega}^{\mathrm{e}}
$$

where $\boldsymbol{\Omega}^{\mathrm{p}}$ and $\boldsymbol{\Omega}^{\mathrm{e}}$ is plastic spin and lattice spin, respectively. The plastic spin $\boldsymbol{\Omega}^{\mathrm{P}}$ is induced by crystal slip on slip plane along slip direction, during which the crystal orientation keeps unchanged. The plastic spin can be calculated according to

$$
\mathbf{\Omega}^{\mathrm{p}}=\sum_{\alpha=1}^{12} \frac{1}{2}\left(\mathbf{s}^{(\alpha)} \cdot \mathbf{m}^{(\alpha)}-\mathbf{m}^{(\alpha)} \cdot \mathbf{s}^{(\alpha)}\right) \dot{\gamma}^{(\alpha)}
$$

Where $\mathbf{s}^{(\alpha)}$ and $\mathbf{m}^{(\alpha)}$ are the slip direction and slip plane normal, respectively. The crystal orientation changes during lattice spin $\left(\boldsymbol{\Omega}^{\mathrm{e}}\right)$. The material spin about RD and ND, i.e., $\boldsymbol{\Omega}_{\mathrm{RD}}$ and $\boldsymbol{\Omega}_{\mathrm{ND}}$, is zero in this study, since the simulation model is 2D. However, the slip systems are oriented in all three dimensions and according to the hardening model, the activation of a slip system is only determined by its resolved shear stress relative to its strength. Thus $\Omega_{\mathrm{RD}}^{\mathrm{p}}$ and/or $\boldsymbol{\Omega}_{\mathrm{ND}}^{\mathrm{p}}$ would develop (Eq.2) if the activated slip systems are not on the RD-ND plane, so lattice have to rotate about RD and ND in the opposite direction to retain zero material spin (Eq.1). This is why crystal rotations about all three axes have been observed in this 2D model. To be computation efficiently, 2D model, like in this study, has been widely used and accurate predictions have been obtained $[6,34,35,42]$ 
by the CPFE model, and no obvious difference in texture evolution can be seen after comparison between 2D and 3D model [43].
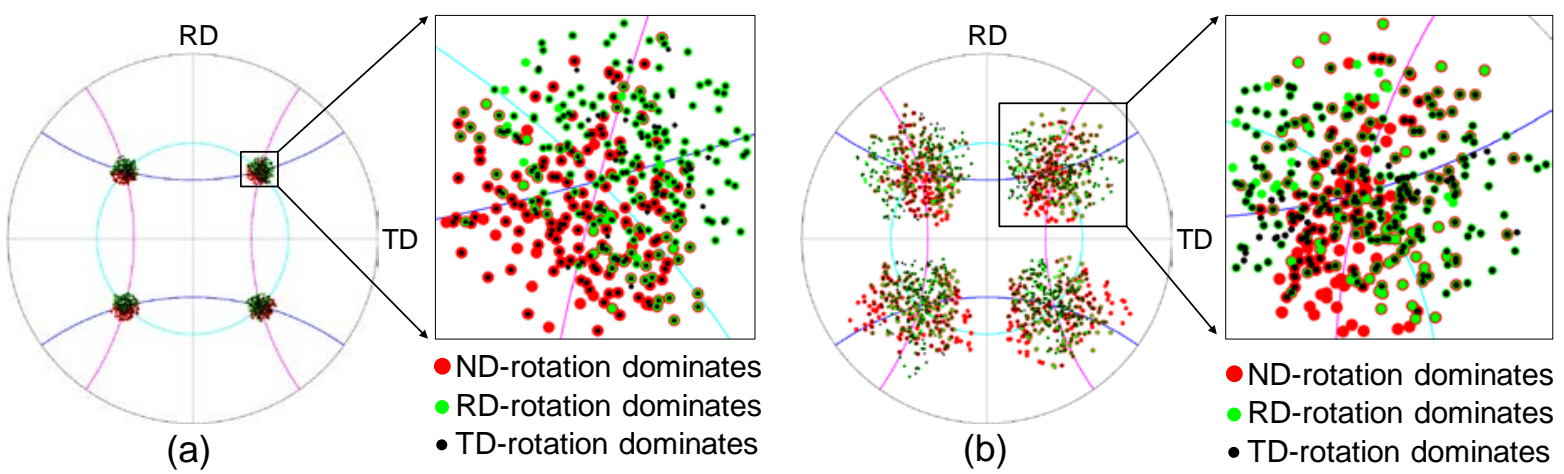

Fig.9. (1 11 1) pole figure of initial orientations and main rotation axes of (a) Dev5, and (b) Dev20.

Grain-interaction is critical to the formation of in-grain and macroscopic texture. As pointed out by Delannay et al [26], the evolution of in-grain texture would greatly change the direction of crystal rotation. For instance, rotations about TD within (near) Cube grains was not observed [26] due to the oversimplification, as suggested by the authors, that one grain was used to represent a grain and thus grain subdivision was not achievable. In this case, the high difference in initial orientations between adjacent grains would produce large difference in plastic spin $\left(\boldsymbol{\Omega}^{\mathrm{P}}\right)$, according to Eq.2. To accommodate the strain incompatibilities $(\boldsymbol{\Omega})$ at grain boundaries, large crystal rotations $\left(\boldsymbol{\Omega}^{\mathrm{e}}\right)$ are required (Eq.1) [25]. Therefore, it seems that the crystal rotation was totally determined by the neighbourhood, but not its initial orientation. This is why the crystal rotation angles are large in the immediate vicinity of grain boundaries (Fig.3d), particularly at triple junctions. Grain interaction originates at grain boundaries and its effect decreases as subdivision propagates into the grain [25]. Therefore, the deformation in the inner region of a grain is basically determined its initial orientation (Fig.3d). In CPFE, no homogenization is in principle assumed, so intra-grain subdivision and intergrain interaction can be accessed. An extensive collection of studies show that taking graininteraction into account provides significantly better texture prediction than that omit it $[22,44]$.

Texture evolution is a pronounced function of the starting texture and the plastic deformation are generally accomplished by a small but dominating set of slip systems [22,34]. Four slip systems with equal Schmid factor are supposed to be activated in Cube [6, 7], which would cause TD-rotation. Though, unlike single crystal, Dev2 is of polycrystal structure, its deformation texture is very similar to exact Cube even up to a $75 \%$ reduction, since the deviation angle and it induced grain-interaction are low. This means that $2^{\circ}$ is not enough to, and cannot cause strong grain-interaction to, alter the activation of slip systems, so Dev2 can be treated as single crystal. However, different deformation behaviours have been observed in Dev5. Though TD-rotation is still dominant, a close inspection revealed that $28.4 \%$ and $28.3 \%$ of the 11200 elements (not grains) have RD and ND as the main rotation axis (having the largest partitioned rotation angle over the other two components), respectively. The initial positions of all elements are shown in Fig.9a and only the main axis of each element is shown. The poles of all elements in a grain overlap, since one grain has about $\sim 30$ elements that are of the same initial orientations. It is clear that there are only a few grains that all elements in them have the identical main rotation axis, i.e., in a single colour. Generally speaking, these grains are of large initial deviation, i.e., far away from Cube, where the initial orientation plays 
a ruling role. Most grains are represented by two colours, which means grain subdivision occurred in them, since portions of a grain rotated into different directions. Different combinations of rotation axis have been observed in Fig.9a, which corresponds to the experimental observation in 5TD, 5ND and 5RD (Fig.4) that the direction of initial rotation is pivotal. The $\sim 375$ grains, covering the experimental studies of single crystals with specific orientations (like 5TD, 5ND and 5RD), with different initial orientations gave a statistical stability, since various combinations of initial orientations and neighbouring grain configurations can be achieved. To a grain, the effect of initial orientation and grain-interaction varies from grain to grain. As for global texture, TD is the main rotation axis and TD-rotation is greatly higher than the other two components, similar to that in exact Cube.

In Dev20 and Dev45, the activated slip systems are supposed to be different from grain to grain due to the greatly scattered initial orientations and strong grain-interaction. It is believed that the strong grain interaction is responsible for the large crystal rotation angles $\left(>15^{\circ}\right)$ in Dev20 and Dev45 (Fig.5d), since the crystal rotation angle is usually below $15^{\circ}$ for deformed single crystals that have no grain-interaction [11, 45-47], i.e., uniform initial orientations, and moreover, the crystal rotation angles in the inner grain, caused by initial orientation, is also at the level of $15^{\circ}$ (Fig.3d). In Dev20, $29.3 \%, 34 \%$ and $36.7 \%$ of the 11200 elements have RD, ND and TD as the main rotation axis (Fig.9b), respectively, and the crystal rotations about the three axes are comparable (Fig.5c). However, after tracing the deformation history it was found that TD-rotation evolved first, corresponding to the finding in Ref. [27]. Strong grain-interaction resulted in large rotation angles (Fig.5d) and accelerate the formation of rolling texture. The sharp texture predicted by the Taylor model and self-consistent model was caused by the strong grain-interaction [22]. The rolling texture in Dev20 after the second pass (Fig.6) is still weaker than that in Dev45 after the first pass (Fig.4) because the grain-interaction in the former is lower than that in the later. Moreover, in-grain subdivision would initiate subgraininteraction, since the orientations between adjacent elements are different (Fig.3d), which in turn would accelerate grain subdivision. Increasing the number of elements in grains would be beneficial to reducing grain interaction [22]. The number of elements in a grain ( 30) is still limited in this study, which is seriously constrained by the computation capability.

\section{Conclusions}

Four rolling models with different initial orientation scatter from exact Cube were conducted by CPFE and the following can be concluded.

1. A CPFE model was developed to investigate the coupled effect of initial orientation scatter and grain-interaction. The prediction has been validated by the experimental observations. The intraand inter-grain deformation are accessible in the CPFE, which is critical to the accurate predictions.

2. For the deviation angle of $2^{\circ}$, TD-rotation is greatly dominant up to a $75 \%$ reduction, and the prediction in this case is close to that observed in exact Cube. The effect of deviation angles of $2^{\circ}$ is trivial. TD-rotation dominates in Dev5 at a 50\% reduction, and then non-TD-rotation evolved as the reduction increased to $75 \%$. The effect of initial orientation and grain-interaction varies from grain to grain, but the global texture in Dev5 is still close to exact Cube at a $50 \%$ reduction. 
3. Crystal rotations about all three axes are comparably large in Dev20 and Dev45 at a 50\% reduction and rolling texture formed. However, the rotation rate decreased in the second pass as the orientations approached stable positions.

4. The effect of grain orientation and grain-interaction to in-grain subdivision was studied by regenerating the orientations of the neighbouring grains. Crystal rotation behaviours in an individual grain are basically determined by its initial orientation and influenced by neighbouring grains. The effect of grain-interaction is limited to the elements close to grain boundaries.

\section{Acknowledgements}

The simulation was performed on the HPC cluster of the University of Wollongong.

\section{References}

1. Oddershede, J., et al., Deformation-induced orientation spread in individual bulk grains of an interstitial-free steel. Acta Materialia, 2015. 85: p. 301-313.

2. Rezvanian, O., M.A. Zikry, and A.M. Rajendran, Microstructural modeling of grain subdivision and large strain inhomogeneous deformation modes in f.c.c. crystalline materials. Mechanics of Materials, 2006. 38(12): p. 1159-1169.

3. Van Houtte, P., et al., Deformation texture prediction: From the Taylor model to the advanced Lamel model. International Journal of Plasticity, 2005. 21(3): p. 589-624.

4. Liu, Q., J. Wert, and N. Hansen, Location-dependent lattice rotation and shear strain in rolled aluminum single crystals of cube and Goss orientations. Acta materialia, 2000. 48(17): p. 4267-4279.

5. $\quad \mathrm{Si}$, L.Y., et al., Simulation of rolling behaviour of cubic oriented al single crystal with crystal plasticity FEM. Journal of Materials Processing Technology, 2008. 201(1-3): p. 79-84.

6. Deng, G.Y., et al., Influence of cold rolling reduction on the deformation behaviour and crystallographic orientation development. Computational Materials Science, 2013.

7. Liu, Q. and N. Hansen, Macroscopic and microscopic subdivision of a cold-rolled aluminium single crystal of cubic orientation. Proceedings of the Royal Society A: Mathematical, Physical and Engineering Sciences, 1998. 454(1978): p. 2555-2591.

8. Kashihara, K. and H. Inagaki, Effect of external constraint and deviation from ideal orientation on development of rolling texture in pure aluminum single crystal having $\{100\}$ $<001>$ orientation, in Ceramic Transactions. 2008. p. 453-460.

9. Mukhopadhyay, P. and S. Badirujjaman, Relative stability of cube orientation in single crystal aluminium during deformation. Transactions of the Indian Institute of Metals, 2012. 65(4): p. 343-353.

10. Kashihara, K. and H. Inagaki, Rolling textures in aluminum single crystal deviated by 5 degrees about rolling direction from (001) [100] orientation. Materials Transactions, 2007. 48(8): p. 1986-1991.

11. Kashihara, K., et al., Change in crystal orientations of a \{100\} $\{001\rangle$ pure aluminum single crystal during accumulative roll bonding. Materials Transactions, 2011. 52(5): p. 825-829.

12. Kashihara, K., D. Terada, and N. Tsuji, Formation of dillamore orientation during accumulative roll bonding of $\{001\}<100>$ aluminum single crystal. Keikinzoku/Journal of Japan Institute of Light Metals, 2014. 64(3): p. 93-97.

13. Raabe, D., Z. Zhao, and F. Roters, Study on the orientational stability of cube-oriented FCC crystals under plane strain by use of a texture component crystal plasticity finite element method. Scripta Materialia, 2004. 50(7): p. 1085-1090. 
14. Becker, R., et al., Analysis of an aluminum single crystal with unstable initial orientation (001) [110] in channel die compression. Metallurgical Transactions A, 1991. 22(1): p. 45-58.

15. Mishin, O.V., B. Bay, and D. Juul Jensen, Through-thickness texture gradients in cold-rolled aluminum. Metallurgical and Materials Transactions A: Physical Metallurgy and Materials Science, 2000. 31(6): p. 1653-1662.

16. Engler, O., M.Y. Huh, and C.N. Tomé, A study of through-thickness texture gradients in rolled sheets. Metallurgical and Materials Transactions A: Physical Metallurgy and Materials Science, 2000. 31(9): p. 2299-2315.

17. Bertinetti, M.A., C.D. Schwindt, and J.W. Signorelli, Effect of the cube orientation on formability for FCC materials: A detailed comparison between full-constraint and selfconsistent predictions. International Journal of Mechanical Sciences, 2014. 87: p. 200-217.

18. Signorelli, J.W. and M.A. Bertinetti, On the role of constitutive model in the forming limit of FCC sheet metal with cube orientations. International Journal of Mechanical Sciences, 2009. 51(6): p. 473-480.

19. Liu, B., et al., Comparison of finite element and fast Fourier transform crystal plasticity solvers for texture prediction. Modelling and Simulation in Materials Science and Engineering, 2010. 18(8): p. 085005.

20. Khan, A.S., et al., Strain rate effect of high purity aluminum single crystals: Experiments and simulations. International Journal of Plasticity, 2015. 67: p. 39-52.

21. Wenk, H.R. and P. Van Houtte, Texture and anisotropy. Reports on Progress in Physics, 2004. 67(8): p. 1367-1428.

22. Raabe, D., Z. Zhao, and W. Mao, On the dependence of in-grain subdivision and deformation texture of aluminum on grain interaction. Acta materialia, 2002. 50(17): p. 4379-4394.

23. Delannay, L., et al., Measurement of In-Grain Orientation Gradients by EBSD and Comparison with Finite Element Results. Advanced Engineering Materials, 2003. 5(8): p. 597-600.

24. Raabe, D., et al., Theory of orientation gradients in plastically strained crystals. Acta Materialia, 2002. 50(2): p. 421-440.

25. Kanjarla, A.K., L. Delannay, and P. Van Houtte, Finite Element Study of Intragrain Plastic Heterogeneity near a Triple Junction. Metallurgical and Materials Transactions A, 2011. 42(3): p. 660-668.

26. Delannay, L., S.R. Kalidindi, and P. Van Houtte, Quantitative prediction of textures in aluminium cold rolled to moderate strains. Materials Science and Engineering: A, 2002. 336(1): p. 233-244.

27. Quey, R., J.H. Driver, and P.R. Dawson, Intra-grain orientation distributions in hot-deformed aluminium: Orientation dependence and relation to deformation mechanisms. Journal of the Mechanics and Physics of Solids, 2015. 84: p. 506-527.

28. Asaro, R.J., CRYSTAL PLASTICITY. Journal of Applied Mechanics, Transactions ASME, 1983. 50(4 b): p. 921-934.

29. Peirce, D., R.J. Asaro, and A. Needleman, An analysis of nonuniform and localized deformation in ductile single crystals. Acta Metallurgica, 1982. 30(6): p. 1087-1119.

30. Peirce, D., R.J. Asaro, and A. Needleman, Material rate dependence and localized deformation in crystalline solids. Acta Metallurgica, 1983. 31(12): p. 1951-1976.

31. Wu, T.-Y., J. L.Bassani, and C. Laird, Latent Hardening in Single Crystals I. Theory and Experiments. Proceedings of the Royal Society A: Mathematical, Physical and Engineering Sciences, 1991. 435: p. 20.

32. L.Bassani, J. and T.-Y. Wu, Latent Hardening in Single Crystals II. Analytical Characterization and Predictions. Proceedings of the Royal Society A: Mathematical, Physical and Engineering Sciences, 1991. 435: p. 21.

33. Lin, G. and K.S. Havner, A comparative study of hardening theories in torsion using the Taylor polycrystal model. International Journal of Plasticity, 1996. 12(5): p. 695-718. 
34. Lu, C., et al., Crystal plasticity modeling of texture evolution and heterogeneity in equal channel angular pressing of aluminum single crystal. Acta materialia, 2011. 59(9): p. 35813592.

35. Si, L.Y., et al., Simulation of rolling behaviour of cubic oriented al single crystal with crystal plasticity FEM. Journal of Materials Processing Technology, 2008. 201(1): p. 79-84.

36. Inoue, T. and N. Tsuji, Quantification of strain in accumulative roll-bonding under unlubricated condition by finite element analysis. Computational Materials Science, 2009. 46(1): p. 261-266.

37. Wert, J.A., Q. Liu, and N. Hansen, Dislocation boundary formation in a cold-rolled cubeoriented Al single crystal. Acta materialia, 1997. 45(6): p. 2565-2576.

38. Knezevic, M., et al., Texture evolution in two-phase $\mathrm{Zr} / \mathrm{Nb}$ lamellar composites during accumulative roll bonding. International Journal of Plasticity, 2014. 57: p. 16-28.

39. Mayeur, J.R., et al., A crystal plasticity study of heterophase interface character stability of $\mathrm{Cu} / \mathrm{Nb}$ bicrystals. International Journal of Plasticity, 2013. 48(0): p. 72-91.

40. Wert, J.A., Macroscopic crystal rotation patterns in rolled aluminium single crystals. Acta materialia, 2002. 50(12): p. 3125-3139.

41. Rossiter, J., et al., A new crystal plasticity scheme for explicit time integration codes to simulate deformation in 3D microstructures: Effects of strain path, strain rate and thermal softening on localized deformation in the aluminum alloy 5754 during simple shear. International Journal of Plasticity, 2010. 26(12): p. 1702-1725.

42. Mayeur, J.R., et al., A crystal plasticity study of heterophase interface character stability of $\mathrm{Cu} / \mathrm{Nb}$ bicrystals. International Journal of Plasticity, 2013. 48: p. 72-91.

43. Knezevic, M., et al., Three dimensional predictions of grain scale plasticity and grain boundaries using crystal plasticity finite element models. Computer Methods in Applied Mechanics and Engineering, 2014. 277: p. 239-259.

44. Thorning, C., M.A.J. Somers, and J.A. Wert, Grain interaction effects in polycrystalline Cu. Materials Science and Engineering: A, 2005. 397(1): p. 215-228.

45. Yoshida, A., Y. Miyajima, and S. Onaka, Evolution of the spread of crystal orientation with plastic deformation in a cold-rolled Cu single crystal. Journal of Materials Science, 2014. 49(5): p. 2013-2017.

46. Kashihara, K., et al., Stability of $\{4411\}$ 〈1 111 8〉 orientation in a $\{123\} \quad\langle 634\rangle$ aluminum single crystal processed by accumulative roll bonding. Materials Transactions, 2014. 55(11): p. 1656-1661.

47. Kashihara, K., et al., Texture evolution in $\{112\}<111>$ aluminum single crystals processed by severe plastic deformation. Materials Characterization, 2012. 75: p. 129-137. 